\title{
Texture 4 zero Fritzsch-like lepton mass matrices
}

\author{
Gulsheen Ahuja ${ }^{1}$, Sanjeev Kumar², Monika Randhawa ${ }^{3}$, \\ Manmohan Gupta ${ }^{1, *}$ S. Dev ${ }^{2}$ \\ ${ }^{1}$ Department of Physics, Centre of Advanced Study, P.U., Chandigarh, India. \\ ${ }^{2}$ Department of Physics, H.P.U., Shimla, India. \\ ${ }^{3}$ University Institute of Engineering and Technology, P.U., Chandigarh, India.
}

October 15, 2018

\begin{abstract}
For Majorana or Dirac neutrinos, using Fritzsch-like texture 4 zero mass matrices with parallel texture structures for the charged leptons and the Dirac neutrino mass matrix $\left(M_{\nu D}\right)$, detailed predictions for cases pertaining to normal/inverted hierarchy as well as degenerate scenarios of neutrino masses have been carried out. The inverted hierarchy as well as degenerate scenarios seem to be ruled out at $3 \sigma$ C.L. for both Majorana and Dirac neutrinos. For normal hierarchy, Jarlskog's rephasing invariant parameter $J$, the CP violating Dirac-like phase $\delta$ and the effective neutrino mass $\left\langle m_{e e}\right\rangle$ have been calculated. For this case, lower limits of $m_{\nu_{1}}$ and $\theta_{13}$ would have implications for the nature of neutrinos.
\end{abstract}

In the last few years, impressive advances have been made in understanding the phenomenology of neutrino oscillations through solar neutrino experiments [1, atmospheric neutrino experiments [2], reactor based experiments [3] and accelerator based experiments [4. At present, one of the key issues in the context of neutrino oscillation phenomenology is to understand the pattern of neutrino masses and mixings which seems to be vastly different from that of quark masses and mixings. In the case of quarks the masses and mixing angles show distinct hierarchy, whereas in the case of neutrinos neither the mixing angles nor the neutrino masses show any distinct hierarchy. In fact, the two mixing angles governing solar and atmospheric neutrino oscillations look to be rather large, the third angle may be very small compared to these. Further, at present there is no consensus about neutrino masses which may show normal/inverted hierarchy or may even be degenerate. Furthermore, the situation becomes complicated when one realizes that neutrino masses are much smaller than charged lepton and quark masses.

*mmgupta@pu.ac.in 
Taking clue from the success of texture specific mass matrices in the context of quarks [5], several attempts [6, 7] have been made to consider similar lepton mass matrices for explaining the pattern of neutrino masses and mixings. In the absence of sufficient amount of data regarding neutrino masses and mixing angles, it would require a very careful scrutiny of all possible textures to find viable structures which are compatible with data and theoretical ideas so that these be kept in mind while formulating mass matrices at the GUT (Grand Unified Theories) scale. In this context, most of the attempts to understand the pattern of neutrino masses and mixings have been carried out using the seesaw mechanism [8] given by

$$
M_{\nu}=-M_{\nu D}^{T}\left(M_{R}\right)^{-1} M_{\nu D},
$$

where $M_{\nu D}$ and $M_{R}$ are respectively the Dirac neutrino mass matrix and the right-handed Majorana neutrino mass matrix. In this context, it should be noted that the predictions are somewhat different, on the one hand when texture is imposed only on $M_{\nu D}$ and $M_{R}$ and on the other hand when $M_{\nu}$ and $M_{\nu D}$ have the same texture by imposing 'texture invariant conditions' [9, 10]. Similarly, as compared to the Majorana case, the predictions are different for Dirac neutrinos even if $M_{\nu D}$ has the same texture as that of $M_{\nu}$.

Texture 4 zero mass matrices are known to explain the pattern of quark masses and mixings [11] as well as are known to be compatible with specific models of GUTs [5, 6] and these could be obtained using considerations of Abelian family symmetries [12]. Assuming normal hierarchy of masses as well as imposing texture 4 zero structure on $M_{\nu D}$ and charged lepton mass matrices, Xing et al. 9] have not only shown the compatibility of these with neutrino oscillation phenomenology but have also shown the seesaw invariance of these structures under certain conditions. Very recently, Matsuda et al. [10] have reiterated the success of texture 4 zero mass matrices in the case of quarks by showing that these mass matrices can accommodate the present value of $\sin 2 \beta$. Also, for normal hierarchy they have shown that texture 4 zero lepton mass matrices can accommodate large values of mixing angle $s_{13}$. In particular, by imposing texture invariant conditions they have shown that $M_{\nu}$ can be texture 2 zero when one assumes Fritzsch-like texture 2 zero structure for $M_{\nu D}, M_{R}$ as well as for charged lepton mass matrix.

In view of the success of texture 4 zero mass matrices in the context of quark mixing phenomenology, it would be interesting to carry out an exhaustive and detailed analysis of these in the case of leptons. In particular, for both Majorana and Dirac neutrinos it would be interesting to investigate the compatibility of texture 4 zero lepton mass matrices with the inverted hierarchy and degenerate scenario of neutrino masses. In the case of Majorana neutrinos due to the absence of any guidelines for $M_{R}$, to keep the number of independent parameters under control, it would perhaps be desirable to keep its structure as simple as possible. It would also be desirable to study the implications of these mass matrices when texture is imposed only on $M_{\nu D}$ and not on $M_{\nu}$.

Considering Fritzsch-like texture 4 zero lepton mass matrices, with neutrinos and charged leptons having parallel texture structures, the purpose of the present communication is to investigate in detail the implications of these for normal/inverted hierarchy as 
well as 'different' degenerate scenarios of neutrino masses. In particular, for the inverted hierarchy and degenerate scenarios, the implications of these structures have been carried out for both Majorana and Dirac neutrinos by exploring the parameter space available to any of the two mixing angles found by giving full variation to other parameters and phases. In the case of normal hierarchy, we have calculated several phenomenological quantities such as Jarlskog's rephasing invariant parameter $J$, the $\mathrm{CP}$ violating Dirac-like phase $\delta$ and the effective neutrino mass $\left\langle m_{e e}\right\rangle$, related to neutrinoless double beta decay $(\beta \beta)_{0 \nu}$.

To begin with, we define the modified Fritzsch-like matrices, e.g.,

$$
M_{l}=\left(\begin{array}{ccc}
0 & A_{l} & 0 \\
A_{l}^{*} & D_{l} & B_{l} \\
0 & B_{l}^{*} & C_{l}
\end{array}\right), \quad M_{\nu D}=\left(\begin{array}{ccc}
0 & A_{\nu} & 0 \\
A_{\nu}^{*} & D_{\nu} & B_{\nu} \\
0 & B_{\nu}^{*} & C_{\nu}
\end{array}\right) \text {, }
$$

$M_{l}$ and $M_{\nu D}$ respectively corresponding to Dirac-like charged lepton and neutrino mass matrices. The above matrices are texture 4 zero type with $D_{l}$ and $D_{\nu}$ being non-zero along with $A_{l(\nu)}=\left|A_{l(\nu)}\right| e^{i \alpha_{l(\nu)}}$ and $B_{l(\nu)}=\left|B_{l(\nu)}\right| e^{i \beta_{l(\nu)}}$, in case these are symmetric then $A_{l(\nu)}^{*}$ and $B_{l(\nu)}^{*}$ should be replaced by $A_{l(\nu)}$ and $B_{l(\nu)}$, as well as $C_{l(\nu)}$ and $D_{l(\nu)}$ should respectively be defined as $C_{l(\nu)}=\left|C_{l(\nu)}\right| e^{i \gamma_{l(\nu)}}$ and $D_{l(\nu)}=\left|D_{l(\nu)}\right| e^{i \omega_{l(\nu)}}$.

To fix the notations and conventions as well as to facilitate the understanding of inverted hierarchy case and its relationship to the normal hierarchy case, we detail the formalism connecting the mass matrix to the neutrino mixing matrix. The mass matrices $M_{l}$ and $M_{\nu D}$ given in equation (2), for Hermitian as well as symmetric case, can be exactly diagonalized. Details of Hermitian case can be looked up in our earlier work [13], the symmetric case can similarly be worked out. To facilitate diagonalization, the mass matrix $M_{k}$, where $k=l, \nu D$, can be expressed as

$$
M_{k}=Q_{k} M_{k}^{r} P_{k}
$$

or

$$
M_{k}^{r}=Q_{k}^{\dagger} M_{k} P_{k}^{\dagger}
$$

where $M_{k}^{r}$ is a real symmetric matrix with real eigenvalues and $Q_{k}$ and $P_{k}$ are diagonal phase matrices. For the hermitian case $Q_{k}=P_{k}^{\dagger}$, whereas for the symmetric case under certain conditions $Q_{k}=P_{k}$. In general, the real matrix $M_{k}^{r}$ is diagonalized by the orthogonal transformation $O_{k}$, e.g.,

$$
M_{k}^{\text {diag }}=O_{k}^{T} M_{k}^{r} O_{k},
$$

which on using equation (41) can be rewritten as

$$
M_{k}^{\text {diag }}=O_{k}^{T} Q_{k}^{\dagger} M_{k} P_{k}^{\dagger} O_{k}
$$

To facilitate the construction of diagonalization transformations for different hierarchies, we introduce a diagonal phase matrix $\xi_{k}$ defined as $\operatorname{diag}\left(1, e^{i \pi}, 1\right)$ for the case of normal 
hierarchy and $\operatorname{as} \operatorname{diag}\left(1, e^{i \pi}, e^{i \pi}\right)$ for the case of inverted hierarchy. Equation (6) can now be written as

$$
\xi_{k} M_{k}^{\text {diag }}=O_{k}^{T} Q_{k}^{\dagger} M_{k} P_{k}^{\dagger} O_{k},
$$

which can also be expressed as

$$
M_{k}^{\text {diag }}=\xi_{k}^{\dagger} O_{k}^{T} Q_{k}^{\dagger} M_{k} P_{k}^{\dagger} O_{k}
$$

Making use of the fact that $O_{k}^{*}=O_{k}$ it can be further expressed as

$$
M_{k}^{\text {diag }}=\left(Q_{k} O_{k} \xi_{k}\right)^{\dagger} M_{k}\left(P_{k}^{\dagger} O_{k}\right)
$$

from which one gets

$$
M_{k}=Q_{k} O_{k} \xi_{k} M_{k}^{\text {diag }} O_{k}^{T} P_{k}
$$

The case of leptons is fairly straight forward, for the neutrinos the diagonalizing transformation is hierarchy specific as well as requires some fine tuning of the phases of the right handed neutrino mass matrix $M_{R}$. To clarify this point further, in analogy with equation (10), we can express $M_{\nu D}$ as

$$
M_{\nu D}=Q_{\nu D} O_{\nu D} \xi_{\nu D} M_{\nu D}^{\text {diag }} O_{\nu D}^{T} P_{\nu D}
$$

Substituting the above value of $M_{\nu D}$ in equation (11) one obtains

$$
M_{\nu}=-\left(Q_{\nu D} O_{\nu D} \xi_{\nu D} M_{\nu D}^{\operatorname{diag}} O_{\nu D}^{T} P_{\nu D}\right)^{T}\left(M_{R}\right)^{-1}\left(Q_{\nu D} O_{\nu D} \xi_{\nu D} M_{\nu D}^{\text {diag }} O_{\nu D}^{T} P_{\nu D}\right) .
$$

On using $P_{\nu D}^{T}=P_{\nu D}$, the above equation can further be written as

$$
M_{\nu}=-P_{\nu D} O_{\nu D} M_{\nu D}^{\text {diag }} \xi_{\nu D} O_{\nu D}^{T} Q_{\nu D}^{T}\left(M_{R}\right)^{-1} Q_{\nu D} O_{\nu D} \xi_{\nu D} M_{\nu D}^{\text {diag }} O_{\nu D}^{T} P_{\nu D}
$$

Assuming fine tuning, the phase matrices $Q_{\nu D}^{T}$ and $Q_{\nu D}$ along with $-M_{R}$ can be taken as $m_{R} \operatorname{diag}(1,1,1)$ as well as using the unitarity of $\xi_{\nu D}$ and orthogonality of $O_{\nu D}$, the above equation can be expressed as

$$
M_{\nu}=P_{\nu D} O_{\nu D} \frac{\left(M_{\nu D}^{\text {diag }}\right)^{2}}{\left(m_{R}\right)^{-1}} O_{\nu D}^{T} P_{\nu D} .
$$

The lepton mixing matrix, obtained from the matrices used for diagonalizing the mass matrices $M_{l}$ and $M_{\nu}$, is expressed as

$$
U=\left(Q_{l} O_{l} \xi_{l}\right)^{\dagger}\left(P_{\nu D} O_{\nu D}\right)
$$

Eliminating the phase matrix $\xi_{l}$ by redefinition of the charged lepton phases, the above equation becomes

$$
U=O_{l}^{\dagger} Q_{l} P_{\nu D} O_{\nu D}
$$

where $Q_{l} P_{\nu D}$, without loss of generality, can be taken as $\left(e^{i \phi_{1}}, 1, e^{i \phi_{2}}\right), \phi_{1}$ and $\phi_{2}$ being 
related to the phases of mass matrices and can be treated as free parameters.

To understand the relationship between diagonalizing transformations for different hierarchies of neutrino masses as well as their relationship with the charged lepton case, we first consider the general diagonalizing transformation $O_{k}$, whose elements can be written as

$$
\begin{aligned}
& O_{k}(11)=\sqrt{\frac{m_{2} m_{3}\left(m_{3}+m_{2}-D_{l(\nu)}\right)}{\left(m_{1}+m_{2}+m_{3}-D_{l(\nu)}\right)\left(m_{1}-m_{3}\right)\left(m_{1}-m_{2}\right)}} \\
& O_{k}(12)=\sqrt{\frac{m_{1} m_{3}\left(m_{1}+m_{3}-D_{l(\nu)}\right)}{\left(m_{1}+m_{2}+m_{3}-D_{l(\nu)}\right)\left(m_{2}-m_{3}\right)\left(m_{2}-m_{1}\right)}} \\
& O_{k}(13)=\sqrt{\frac{m_{1} m_{2}\left(m_{2}+m_{1}-D_{l(\nu)}\right)}{\left(m_{1}+m_{2}+m_{3}-D_{l(\nu)}\right)\left(m_{3}-m_{2}\right)\left(m_{3}-m_{1}\right)}} \\
& O_{k}(21)=\sqrt{\frac{m_{1}\left(m_{3}+m_{2}-D_{l(\nu)}\right)}{\left(m_{3}-m_{1}\right)\left(m_{1}-m_{2}\right)}} \\
& O_{k}(22)=\sqrt{\frac{m_{2}\left(m_{3}+m_{1}-D_{l(\nu)}\right)}{\left(m_{2}-m_{3}\right)\left(m_{2}-m_{1}\right)}} \\
& O_{k}(23)=\sqrt{\frac{m_{3}\left(m_{2}+m_{1}-D_{l(\nu)}\right)}{\left(m_{2}-m_{3}\right)\left(m_{3}-m_{1}\right)}} \\
& O_{k}(31)=\sqrt{\frac{m_{1}\left(m_{1}+m_{2}-D_{l(\nu)}\right)\left(m_{1}-m_{3}\right)\left(m_{1}+m_{3}-D_{l(\nu)}\right)}{\left(m_{1}-m_{3}\right)^{2}\left(m_{1}-m_{2}\right)\left(m_{1}+m_{2}+m_{3}-D_{l(\nu)}\right)}} \\
& O_{k}(32)=\sqrt{\frac{m_{2}\left(D_{l(\nu)}-m_{1}-m_{2}\right)\left(D_{l(\nu)}-m_{2}-m_{3}\right)}{\left(m_{1}+m_{2}+m_{3}-D_{l(\nu)}\right)\left(m_{2}-m_{3}\right)\left(m_{2}-m_{1}\right)}} \\
& O_{k}(33)=\sqrt{\frac{m_{3}\left(D_{l(\nu)}-m_{2}-m_{3}\right)\left(D_{l(\nu)}-m_{1}-m_{3}\right)}{\left(m_{1}+m_{2}+m_{3}-D_{l(\nu)}\right)\left(m_{3}-m_{1}\right)\left(m_{3}-m_{2}\right)}},
\end{aligned}
$$

where $m_{1}, m_{2}, m_{3}$ are eigenvalues of $M_{k}$. In the case of charged leptons, because of the hierarchy $m_{e} \ll m_{\mu} \ll m_{\tau}$, the mass eigenstates can be approximated respectively to the flavor eigenstates as has been considered by several authors [6, 14]. Using the approximation, $m_{l 1} \simeq m_{e}, m_{l 2} \simeq m_{\mu}$ and $m_{l 3} \simeq m_{\tau}$, the first element of the matrix $O_{l}$ can be obtained from the corresponding element of equation (17) by replacing $m_{1}, m_{2}$, $m_{3}$ by $m_{e},-m_{\mu}, m_{\tau}$, e.g.,

$$
O_{l}(11)=\sqrt{\frac{m_{\mu} m_{\tau}\left(m_{\tau}-m_{\mu}-D_{l}\right)}{\left(m_{e}-m_{\mu}+m_{\tau}-D_{l}\right)\left(m_{\tau}-m_{e}\right)\left(m_{e}+m_{\mu}\right)}} .
$$

For normal hierarchy defined as $m_{\nu_{1}}<m_{\nu_{2}} \ll m_{\nu_{3}}$, as well as for the corresponding degenerate case given by $m_{\nu_{1}} \lesssim m_{\nu_{2}} \sim m_{\nu_{3}}$, equation (17) can also be used to obtain the 
first element of diagonalizing transformation for Majorana neutrinos. By replacing $m_{1}$, $m_{2}, m_{3}$ by $\sqrt{m_{\nu 1} m_{R}},-\sqrt{m_{\nu 2} m_{R}}, \sqrt{m_{\nu 3} m_{R}}$ in the equation, we get

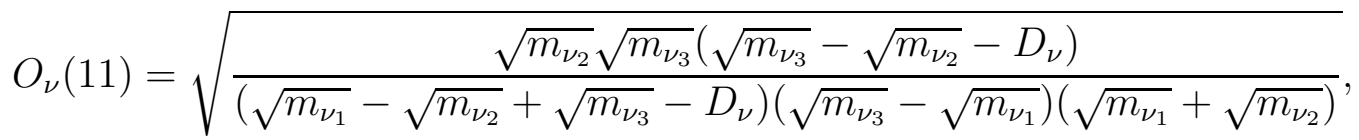

where $m_{\nu_{1}}, m_{\nu_{2}}$ and $m_{\nu_{3}}$ are neutrino masses. The parameter $D_{\nu}$ is to be divided by $\sqrt{m_{R}}$, however as $D_{\nu}$ is arbitrary therefore we retain it as it is.

In the same manner, one can obtain the elements of diagonalizing transformation for the inverted hierarchy case defined as $m_{\nu_{3}} \ll m_{\nu_{1}}<m_{\nu_{2}}$ as well as for the corresponding degenerate case given by $m_{\nu_{3}} \sim m_{\nu_{1}} \lesssim m_{\nu_{2}}$. By replacing $m_{1}, m_{2}, m_{3}$ in equation (17) with $\sqrt{m_{\nu_{1}} m_{R}},-\sqrt{m_{\nu_{2}} m_{R}},-\sqrt{m_{\nu_{3}} m_{R}}$, we obtain

$$
O_{\nu}(11)=\sqrt{\frac{\sqrt{m_{\nu_{2}}} \sqrt{m_{\nu_{3}}}\left(D_{\nu}+\sqrt{m_{\nu_{2}}}+\sqrt{m_{\nu_{3}}}\right)}{\left(-\sqrt{m_{\nu_{1}}}+\sqrt{m_{\nu_{2}}}+\sqrt{m_{\nu_{3}}}+D_{\nu}\right)\left(\sqrt{m_{\nu_{1}}}+\sqrt{m_{\nu_{3}}}\right)\left(\sqrt{m_{\nu_{1}}}+\sqrt{m_{\nu_{2}}}\right)} .}
$$

The other elements of diagonalizing transformations in the case of neutrinos as well as charged leptons can similarly be found. The above formalism has been presented for Majorana neutrinos, for the Dirac case the mixing matrix can easily be derived from diagonalizing transformation of $M_{l}$ and $M_{\nu D}$.

It may be of interest to mention that in the case of normal hierarchy, the formulation of Matsuda et al. [10] can easily be obtained from the present case. For example, their element $O_{11}$ can be obtained from our $O_{k}(11)$ by replacing ' $d_{f}$ ' by ' $m_{1}+m_{2}+m_{3}+D_{l(\nu)}$ ', similarly their other elements can be derived from the elements of our $O_{k}$. Further, it should also be noted that they have treated the $3 \times 3$ element of the mass matrix as free parameter whereas in the present case we have treated $2 \times 2$ element as a free parameter. Furthermore, we have not put any conditions so as to obtain a particular texture for $M_{\nu}$.

Before discussing the results, we would like to mention some of the details pertaining to various inputs. For the purpose of our analysis we have used the results obtained by a recent global analysis carried out by Valle [15], incorporating solar [1], atmospheric [2], reactor [3] and accelerator based experiments [4]. The $3 \sigma$ values of the neutrino mass and mixing parameters so obtained are

$$
\begin{gathered}
\Delta m_{12}^{2}=(7.1-8.9) \times 10^{-5} \mathrm{eV}^{2}, \quad \Delta \mathrm{m}_{23}^{2}=(2.0-3.2) \times 10^{-3} \mathrm{eV}^{2}, \\
\sin ^{2} \theta_{12}=0.24-0.40, \quad \sin ^{2} \theta_{23}=0.34-0.68, \quad \sin ^{2} \theta_{13} \leq 0.040 .
\end{gathered}
$$

These values are quite compatible with those obtained very recently by a global analysis carried out by Garcia and Maltoni [16]. It may be mentioned that the present upper bound on $\sin \theta_{13}$ is somewhat lower than the CHOOZ bound [17]. Further, for the purpose of calculations, we have taken the lightest neutrino mass, the phases $\phi_{1}, \phi_{2}$ and $D_{l, \nu}$ as free parameters, the other two masses are constrained by $\Delta m_{12}^{2}=m_{\nu_{2}}^{2}-m_{\nu_{1}}^{2}$ and $\Delta m_{23}^{2}=m_{\nu_{3}}^{2}-m_{\nu_{2}}^{2}$ in the normal hierarchy case and by $\Delta m_{23}^{2}=m_{\nu_{2}}^{2}-m_{\nu_{3}}^{2}$ in the 
inverted hierarchy case. It may be noted that lightest neutrino mass corresponds to $m_{\nu_{1}}$ for the normal hierarchy case and to $m_{\nu_{3}}$ for the inverted hierarchy case. For all the three hierarchies, the explored range of the lightest neutrino mass is taken to be $10^{-8} \mathrm{eV}-10^{-1} \mathrm{eV}$, our conclusions remain unaffected even if the range is extended further. In the absence of any constraint on the phases, $\phi_{1}$ and $\phi_{2}$ have been given full variation from 0 to $2 \pi$. Although $D_{l, \nu}$ are free parameters, however, they have been constrained such that diagonalizing transformations $O_{l}$ and $O_{\nu}$ always remain real. This implies, for leptons $-\left(m_{l_{2}}-m_{l_{1}}\right)<D_{l}<\left(m_{l_{3}}+m_{l_{2}}\right)$, for Dirac neutrinos $-\left(m_{\nu_{2}}-m_{\nu_{1}}\right)<D_{\nu}<\left(m_{\nu_{3}}-m_{\nu_{2}}\right)$ for normal hierarchy and $-\left(m_{\nu_{2}}-m_{\nu_{1}}\right)<D_{\nu}<\left(m_{\nu_{1}}-m_{\nu_{3}}\right)$ for inverted hierarchy. Similarly, for Majorana neutrinos $-\left(\sqrt{m_{\nu_{2}}}-\sqrt{m_{\nu_{1}}}\right)<D_{\nu}<\left(\sqrt{m_{\nu_{3}}}-\sqrt{m_{\nu_{2}}}\right)$ for normal hierarchy and $-\left(\sqrt{m_{\nu_{2}}}-\sqrt{m_{\nu_{1}}}\right)<D_{\nu}<\left(\sqrt{m_{\nu_{1}}}-\sqrt{m_{\nu_{3}}}\right)$ for inverted hierarchy. The calculations pertaining to the case when charged leptons are in flavor basis can easily be deduced from the above calculations for both Majorana and Dirac neutrinos.

Considering Majorana or Dirac neutrinos, we have carried out detailed calculations pertaining to texture 4 zero lepton mass matrices for the possibilities of neutrino masses having normal/inverted hierarchy or being degenerate. To begin with, we consider the inverted hierarchy case for both types of neutrinos. In this context, it may be mentioned that for both the possibilities texture is imposed only on $M_{\nu D}$, with no such restriction on $M_{\nu}$ for the Majorana case. In figures $1 \mathrm{a}, 1 \mathrm{~b}$ and $1 \mathrm{c}$, for Majorana neutrinos we have plotted the parameter space corresponding to any of the two mixing angles by constraining the third angle by its values given in equation (22) while giving full allowed variation to other parameters. Also included in the figures are blank rectangular regions indicating the experimentally allowed $3 \sigma$ region of the plotted angles. Interestingly, a general look at these figures reveals that the case of inverted hierarchy seems to be ruled out. A closer look at these figures brings out several interesting points. From figure 1a showing the plot of angles $\theta_{12}$ versus $\theta_{23}$, one can immediately conclude that the plotted parameter space includes the experimentally allowed range of $\theta_{23}=35.7^{\circ}-55.6^{\circ}$, however it excludes the experimentally allowed range of $\theta_{12}=29.3^{\circ}-39.25^{\circ}$. This clearly indicates that at $3 \sigma$ C.L. inverted hierarchy is not viable. It may be noted that while plotting this figure, $\theta_{13}$ is restricted by the bound given in equation (22), while $\Delta m_{12}^{2}$ and $\Delta m_{23}^{2}$ are constrained by the experimental limits given in equation (21). It may also be mentioned that although the $3 \sigma$ upper limit of angle $\theta_{12}$ is not included in the plotted parameter space, yet it lies very near to the boundary, therefore the above conclusions needs to be checked further.

The conclusions arrived above can be further checked from figures $1 \mathrm{~b}$ and $1 \mathrm{c}$ wherein we have plotted $\theta_{12}$ versus $\theta_{13}$ and $\theta_{23}$ versus $\theta_{13}$ respectively by constraining angles $\theta_{23}$ and $\theta_{12}$. Both the figures indicate that the plotted parameter space does not include simultaneously the experimental bounds of the plotted angles, e.g., $\theta_{12}$ in the case of figure $1 \mathrm{~b}$ and $\theta_{13}$ in figure 1c. Here it needs to be mentioned that similar to figure $1 \mathrm{a}$, in figure $1 \mathrm{~b}$ also the $3 \sigma$ upper limit of angle $\theta_{12}$ lies very near to the boundary of the plotted parameter space, however in figure $1 \mathrm{c}$ the $3 \sigma$ upper limit of angle $\theta_{13}$ is well below the plotted parameter space.

For Dirac neutrinos, again inverted hierarchy seems to be ruled out as can be easily checked from figures $2 \mathrm{a}, 2 \mathrm{~b}$ and $2 \mathrm{c}$, plotted in a manner similar to the Majorana case 
by constraining one mixing angle by its experimental limits and plotting the parameter space for the other two angles. Again, these figures reveal that the plotted parameter space does not overlap with the experimental limits of at least one of the plotted angles, thereby indicating that inverted hierarchy is ruled out at $3 \sigma$ C.L. for Dirac neutrinos as well.

For Majorana or Dirac neutrinos the case of neutrino masses being degenerate, characterized by either $m_{\nu_{1}} \lesssim m_{\nu_{2}} \sim m_{\nu_{3}} \lesssim 0.1 \mathrm{eV}$ or $m_{\nu_{3}} \sim m_{\nu_{1}} \lesssim m_{\nu_{2}} \lesssim 0.1 \mathrm{eV}$ corresponding to normal hierarchy and inverted hierarchy respectively, is again ruled out. Considering degenerate scenario corresponding to inverted hierarchy, figures 1 and 2 can again be used to rule out degenerate scenario at $3 \sigma$ C.L. for Majorana and Dirac neutrinos respectively. It needs to be mentioned that while plotting these figures the range of the lightest neutrino mass is taken to be $10^{-8} \mathrm{eV}-10^{-1} \mathrm{eV}$, which includes the neutrino masses corresponding to degenerate scenario, therefore by discussion similar to the one given for ruling out inverted hierarchy, degenerate scenario of neutrino masses is ruled out as well.

Coming to degenerate scenario corresponding to normal hierarchy, one can easily show that this is ruled out again. To this end, in figure 3 , by giving full variation to other parameters, we have plotted the mixing angle $\theta_{12}$ against the lightest neutrino mass $m_{\nu_{1}}$. Figure 3a corresponds to the case of Majorana neutrinos and figure 3b to the case of Dirac neutrinos. From the figures one can immediately find that the values of $\theta_{12}$ corresponding to $m_{\nu_{1}} \lesssim 0.1 \mathrm{eV}$ lie outside the experimentally allowed range, thereby ruling out degenerate scenario for Majorana as well as Dirac neutrinos at $3 \sigma$ C.L..

The presence of a few isolated points near the experimentally allowed $3 \sigma$ regions shown in figures 1 and 2 may raise doubts about our conclusions. In order to check whether there are any solution points within the experimentally allowed $3 \sigma$ region of plotted angles, we have attempted to obtain a common parameter space pertaining to the three mixing angles simultaneously. Interestingly, we find that all possible cases considered here pertaining to inverted hierarchy and degenerate scenario are again ruled out. It may also be added that in the case when charged leptons are in the flavor basis, one can easily check that inverted hierarchy and degenerate scenarios for the texture 4 zero mass matrices are again ruled out, in agreement with the conclusions of [18]. The results pertaining to this case can easily be derived from our earlier cases.

After ruling out the cases pertaining to inverted hierarchy and degenerate scenario, we now discuss the normal hierarchy cases. For the charged lepton mass matrix $M_{l}$ being Fritzsch-like or in the flavor basis, for Majorana as well as Dirac neutrinos, in table (1) we have presented the viable ranges of neutrino masses, mixing angles $\theta_{12}, \theta_{23}$ and $\theta_{13}$, Jarlskog's rephasing invariant parameter $J$, CP violating phase $\delta$ and effective neutrino mass $\left\langle m_{e e}\right\rangle$. The parameter $J$ can be calculated by using its expression given in [14], whereas $\delta$ can be determined from $J=s_{12} s_{23} s_{13} c_{12} c_{23} c_{13}^{2} \sin \delta$ where $c_{i j}=\cos \theta_{i j}$ and $s_{i j}=\sin \theta_{i j}$, for $i, j=1,2,3$. The effective Majorana mass, related to neutrinoless double beta decay $(\beta \beta)_{0 \nu}$, is given as

$$
\left\langle m_{e e}\right\rangle=m_{\nu_{1}} U_{e 1}^{2}+m_{\nu_{2}} U_{e 2}^{2}+m_{\nu_{3}} U_{e 3}^{2}
$$


Considering the texture 4 zero case when $M_{\nu D}$ and $M_{l}$ both have parallel texture structures, a close look at table (11) reveals several interesting points. For both Dirac or Majorana neutrinos, the viable range of the lightest neutrino mass $m_{\nu_{1}}$ is quite different, in particular the range corresponding to Dirac neutrinos is much wider at both the ends as compared to the Majorana neutrinos. Similar conclusions can be arrived at by studying the implications of the well known mixing angle $\theta_{12}$ on the lightest neutrino mass $m_{\nu_{1}}$ through a closer look at the figures $3 \mathrm{a}$ and $3 \mathrm{~b}$. Therefore, a measurement of $m_{\nu_{1}}$ could have important implications for the nature of neutrinos. Somewhat constrained range of $m_{\nu_{2}}$ for the Majorana case as compared to the Dirac case is also due to the constrained range of $m_{\nu_{1}}$ for the Majorana case. Also, from the table one finds that the lower limit on $\theta_{13}$ for the Dirac case is considerably lower than for the Majorana case, therefore a measurement of $\theta_{13}$ would have important implications for this case. It must be noted that the calculated values of $\left\langle m_{e e}\right\rangle$ are much less compared to the present limits of $\left\langle m_{e e}\right\rangle$ [19], therefore, these do not have any implications for the texture 4 zero cases considered here. However, the future experiments with considerably higher sensitivities, aiming to measure $\left\langle m_{e e}\right\rangle \simeq 3.6 \times 10^{-2} \mathrm{eV}(\mathrm{MOON}[20])$ and $\left\langle m_{e e}\right\rangle \simeq 2.7 \times 10^{-2} \mathrm{eV}(\mathrm{CUORE}[21])$, would have implications on the cases considered here. The different cases of Dirac and Majorana neutrinos do not show any divergence for the ranges of Jarlskog's rephasing invariant parameter .

It may be of interest to construct the Pontecorvo-Maki-Nakagawa-Sakata (PMNS) mixing matrix [22] which for Majorana neutrinos is

$$
U=\left(\begin{array}{lll}
0.7599-0.8701 & 0.4797-0.6294 & 0.0199-0.1994 \\
0.1673-0.5715 & 0.3948-0.7606 & 0.5720-0.8224 \\
0.1854-0.5912 & 0.3549-0.7363 & 0.5540-0.8094
\end{array}\right),
$$

wherein we have given the magnitude of the matrix elements. Similarly, for Dirac neutrinos, the PMNS matrix is

$$
U=\left(\begin{array}{lll}
0.7604-0.9213 & 0.3887-0.6317 & 0.0015-0.1993 \\
0.1475-0.5552 & 0.4049-0.8170 & 0.4154-0.8244 \\
0.1830-0.6022 & 0.3648-0.7441 & 0.5546-0.9095
\end{array}\right)
$$

A general look at the two matrices reveals that the ranges of the matrix elements are more wider in the case of Dirac neutrinos as compared to those in the case of Majorana neutrinos. A comparison of the two matrices shows that the lower limit of the element $U_{\mu 3}$ show an appreciable difference, which seems to be due to the nature of neutrinos, hence, a further precision of $U_{\mu 3}$ would have important implications. Also, it may be mentioned that both the above mentioned matrices are fully compatible with a very recent construction of a mixing matrix by Bjorken et al. [23] assuming democratic trimaximally mixed $\nu_{2}$ mass eigenstate as well as with the one presented by Giunti [24].

For the sake of completion pertaining to normal hierarchy, in table (11) we have also presented the results when charged leptons are in the flavor basis which can be easily deduced from the case when $M_{l}$ is Fritzsch-like. Interestingly, from the table one imme- 
diately finds that in this case both $J$ and $\delta$ are vanishingly small for the wide range of parameters considered here, which can easily be understood by examining the corresponding mixing matrix. Also, the range of angle $\theta_{13}$ is much narrower compared to the case when $M_{l}$ is Fritzsch-like, particularly for the Majorana case the predicted range is very narrow, therefore a measurement of $\theta_{13}$ would have an immediate impact on this case. It may also be added that for the Majorana case, the range of $\theta_{23}$ is compatible only with the lower part of the present admissible range, however for the Dirac case there is no such restriction. These conclusions are broadly in agreement with those of [18].

To summarize, detailed calculations have been carried out for different hierarchies in the case of Fritzsch-like texture 4 zero mass matrices with parallel texture structures for charged leptons and for Dirac neutrino mass matrix $\left(M_{\nu D}\right)$ using latest $3 \sigma$ input values of neutrino mass and mixing parameters. For the inverted hierarchy, pertaining to both Majorana and Dirac neutrinos, parameter space available to any two of the mixing angles has been explored while considering wide ranges of free parameters available. Similarly, the viability of 'different' degenerate scenarios has been examined. Interestingly for both types of neutrinos, inverted hierarchy as well as degenerate scenarios seem to be ruled out at $3 \sigma$ C.L. and hence strongly disfavored. It may also be added that the results when charged leptons are in the flavor basis can easily be deduced from the present calculations and these lead to similar conclusions.

For the normal hierarchy case, several phenomenological quantities such as Jarlskog's rephasing invariant parameter $J$, the $\mathrm{CP}$ violating Dirac-like phase $\delta$ and the effective neutrino mass $\left\langle m_{e e}\right\rangle$ have been calculated. The different cases of Majorana and Dirac neutrinos do not show any divergence for the ranges of $J$ and phase $\delta$. In the case of $m_{\nu_{1}}$ and $\theta_{13}$, the Dirac case seems to accommodate a larger range of these parameters. In particular, a measurement of the lower limits of these parameters would have implications for the nature of neutrinos. Also, the PMNS matrices constructed for Majorana as well as Dirac neutrinos, by giving full variation to the parameters, are compatible with a very recent construction of a mixing matrix by Bjorken et al. [23] assuming democratic trimaximally mixed $\nu_{2}$ mass eigenstate.

\section{Acknowledgements}

M.G. and G.A. would like to thank DAE, BRNS (grant No.2005/37/4/BRNS), India, for financial support. S.K. acknowledges the financial support provided by CSIR, India. M.R. would like to thank the Director, UIET for providing facilities to work.

\section{References}

[1] R. Davis, Prog. Part. Nucl. Phys. 32, (1994) 13 ; B. T. Cleveland et al., Astrophys. J. 496, (1998) 505 ; J.N. Abdurashitov et al., SAGE Collaboration, Phys. Rev. C60, (1999) 055801; C. M. Cattadori, GNO Collaboration, Nucl. Phys. Proc. Suppl. 110, (2002) 311 ; W. Hampel et al., GALLEX Collaboration, Phys. Lett. B447, (1999) 127; S. Fukuda et al., Super-Kamiokande Collaboration, Phys. Lett. B539, (2002) 
179 ; Q. R. Ahmad et al., SNO Collaboration, Phys. Rev. Lett. 89, (2002) 011301; S. N. Ahmad et al., Phys. Rev. Lett. 92, (2004) 181301.

[2] Y. Fukuda et al., SuperKamiokande Collaboration, Phys. Rev. Lett. 81, (1998) 1562; A. Surdo, MACRO Collaboration, Nucl. Phys. Proc. Suppl. 110, (2002) 342; M. Sanchez, Soudan 2 Collaboration, Phys. Rev. D68, (2003) 113004.

[3] K. Eguchi et al., KamLAND Collaboration, Phys. Rev. Lett. 90, (2003) 021802; Phys. Rev. Lett. 94, (2005) 081801.

[4] M. H. Ahn et al., K2K Collaboration, Phys. Rev. Lett. 90, (2003) 041801.

[5] H. Fritzsch, Z. Z. Xing, Prog. Part. Nucl. Phys. 45, (2000) 1, and references therein.

[6] H. Fritzsch, Z. Z. Xing, Prog. Part. Nucl. Phys. J. C36, (2004) 227, and references therein.

[7] K. Kang, S.K. Kang, Phys. Rev. D56, (1997) 1511; H. Nishiura, K. Matsuda, T. Fukuyama, Phys. Rev. D60, (1999) 013006; K. Matsuda, T. Fukuyama, H. Nishiura, Phys. Rev. D61, (2000) 053001; K. Kang, S.K. Kang, C.S. Kim, S.M. Kim, Mod. Phys. Lett. A16, (2001) 2169; C. Giunti, M. Tanimoto, Phys. Rev. D66, (2002) 113006; M. Frigerio, A.Yu. Smirnov, Nucl. Phys. B640, (2002) 233; P.F. Harrison, W.G. Scott, Phys. Lett. B547, (2002) 219; E. Ma, Mod. Phys. Lett. A17, (2002) 2361; E. Ma, Phys. Rev. D66, (2002) 117301; P.H. Frampton, S.L. Glashow, D. Marfatia, Phys. Lett. B 536, (2002) 79; Z.Z. Xing, Phys. Lett. B 530, (2002) 159; Z.Z. Xing, Phys. Lett. B 539, (2002) 85; A. Kageyama, S. Kaneko, N. Simoyama, M. Tanimoto, Phys. Lett. B 538, (2002) 96; W.L. Guo, Z.Z. Xing, hep-ph/0211315; M. Randhawa, G. Ahuja, M. Gupta, Phys. Rev. D65, (2002) 093016; M. Frigerio, A.Yu. Smirnov, Phys. Rev. D67, (2003) 013007; K.S. Babu, E. Ma, J.W.F. Valle, Phys. Lett. B552, (2003) 207; W.L. Guo, Z.Z. Xing, Phys. Rev. D67, (2003) 053002; S. Kaneko, M. Tanimoto, Phys. Lett. B551, (2003) 127; B.R. Desai, D.P. Roy, A.R. Vaucher, Mod.Phys.Lett. A18, (2003) 1355; K. Hasegawa, C.S. Lim, K. Ogure, Phys. Rev. D68, (2003) 053006; M. Honda, S. Kaneko, M. Tanimoto, JHEP 0309, (2003) 028; G. Bhattacharyya, A. Raychaudhuri, A. Sil, Phys. Rev. D67, (2003) 073004; P.F. Harrison, W.G. Scott, Phys. Lett. B594, (2004) 324; Z.Z. Xing, Int. J. Mod. Phys. A19, (2004) 1; M. Bando, S. Kaneko, M. Obara, M. Tanimoto, Phys. Lett. B580, (2004) 229; O.L.G. Peres, A.Yu. Smirnov, Nucl. Phys. B680, (2004) 479; C.H. Albright, Phys. Lett. B599, (2004) 285; J. Ferrandis, S. Pakvasa, Phys. Lett. B603, (2004) 184; S.T. Petcov, W. Rodejohann, Phys. Rev. D71, (2005) 073002; S.S. Masood, S. Nasri, J. Schechter, Phys. Rev. D71, (2005) 093005; R. Dermíšek, S. Raby, Phys. Lett. B622, (2005) 327 ; F. Plentinger, W. Rodejohann, Phys. Lett. B625, (2005) 264.

[8] P. Minkowski, Phys. Lett. B67, (1977) 421; T. Yanagida, in Proc. of Work-shop on Unified Theory and Baryon number in the Universe, eds. O. Sawada, A. Sugamoto, 
KEK, Tsukuba, (1979); M. Gell-Mann, P. Ramond, R. Slansky, in Supergravity, eds P. van Niewenhuizen, D. Z. Freedman (North Holland, Amsterdam 1980); P. Ramond, Sanibel talk, retroprinted as hep-ph/9809459; S. L. Glashow, in Quarks and Leptons, Carg'ese lectures, eds M. Levy, (Plenum, 1980, New York) p. 707; R. N. Mohapatra, G. Sen- janovic, Phys. Rev. Lett. 44, (1980) 912.

[9] Z. Z. Xing, H. Zhang, Phys.Lett. B569, (2003) 30; Z. Z. Xing, S. Zhou, Phys. Lett. B606, (2005) 145.

[10] K. Matsuda, H. Nishiura, Phys. Rev. D74, (2006) 033014, and references therein.

[11] D. Du, Z. Z. Xing, Phys. Rev. D48, (1993) 2349; L.J. Hall, A. Rasin, Phys. Lett. B315, (1993) 164 ; P. Ramond, R. G. Roberts, G. G. Ross, Nucl. Phys. B406, (1993) 19; H. Fritzsch, D. Holtmanspötter, Phys. Lett. B338, (1994) 290; H. Fritzsch, Z.Z. Xing, Phys. Lett. B353, (1995) 114; M. Gupta, P. S. Gill, Pramana 45, (1995) 333; P.S. Gill, M. Gupta, J. Phys. G: Nuc. Part. Phys. 21, (1995) 1; H. Lehmann, C. Newton, T. T. Wu, Phys. Lett. B384, (1996) 249; P. S. Gill, M. Gupta, Jol. Phys. G23, (1997) 1; P. S. Gill, M. Gupta, Phys. Rev. D56, (1997) 3143; Z. Z. Xing, J. Phys. G23, (1997) 1563; T. Kobayashi, Z. Z. Xing, Mod. Phys. Lett. A12, (1997) 561; ibid. Int. J. Mod. Phys. A13, (1998) 2201; P. S. Gill, M. Gupta, Phys. Rev. D57, (1998) 3917; J. L. Chkareuli, C. D. Froggatt, Phys. Lett. B450, (1999) 158; A. Mondragón, E. Rodriguez-Jáuregui, Phys. Rev. D59, (1999) 093009; M. Randhawa, V. Bhatnagar, P. S. Gill, M. Gupta, Phys. Rev. D60, (1999) 051301; G. C. Branco, D. Emmanuel-Costa, R. González Felipe, Phys. Lett. B477, (2000) 147; S. H. Chiu, T. K. Kuo, G. H. Wu, Phys. Rev. D62, (2000) 053014; H. Fritzsch, Z. Z. Xing, Phys. Rev. D61, (2000) 073016; ibid. Phys. Lett. B506, (2001) 109; R. Rosenfeld, J. L. Rosner, Phys. Lett. B516, (2001) 408 ; R. G. Roberts, A. Romanino, G. G. Ross, L. Velasco-Sevilla, Nucl. Phys. B615, (2001) 358; J. L. Chkareuli, C. D. Froggatt, Nucl. Phys. B626, 307 (2002); H. Fritzsch, Z. Z. Xing, Phys. Lett. B555, (2003) 63; Z. Z. Xing, H. Zhang, J. Phys. G30, (2004) 129.

[12] W. Grimus, hep-ph/0511078.

[13] See M. Randhawa et al. from [11].

[14] M. Fukugita, M. Tanimoto, T. Yanagida, Prog. Theor. Phys. 89, (1993) 263; ibid. Phys. Lett. B562, (2003) 273.

[15] J. W. F. Valle, hep-ph/0608101; ibid. hep-ph/0612251.

[16] M.C. Gonzalez-Garcia, M. Maltoni, arXiv:0704.1800v1.

[17] M. Appolonio et al., CHOOZ Collaboration, Eur. Phys. J. C27 (2003) 331.

[18] S. Dev, S. Kumar, S. Verma, S. Gupta, hep-ph/0612102. 
[19] H. V. Klapdor-Kleingrothaus et al., Heidelberg-Moscow collaboration, Eur. Phys. J. A12, (2001) 147; ibid. Phys. Lett. B586, (2004) 198, and references therein.

[20] H. Ejiri, R. Hazama, P. Krastev, N. Kudomi, R. G. H. Robertson, Phys. Rev. Lett. 85, (2000) 2917.

[21] R. Ardito, et al., CUORE Collaboration, hep-ex/0501010; C. Arnaboldi et al., Phys. Rev. Lett. 95, (2005) 142501.

[22] B. Pontecorvo, Zh. Eksp. Teor. Fiz. (JETP) 33, (1957) 549; ibid. 34, (1958) 247; ibid. 53, (1967) 1771; Z. Maki, M. Nakagawa, S. Sakata, Prog. Theor. Phys. 28, (1962) 870 .

[23] J. D. Bjorken, P. F. Harrison, W. G. Scott, Phys. Rev. D74, (2006) 073012.

[24] C. Giunti, hep-ph/0611125. 


\begin{tabular}{|c|cc|cc|}
\hline & \multicolumn{2}{|c|}{$M_{l}$ being Fritzsch-like } & \multicolumn{2}{c|}{$M_{l}$ being in the flavor basis } \\
\hline & Dirac case & Majorana case & Dirac case & Majorana case \\
\hline$m_{\nu_{1}}$ & $5.73 \times 10^{-5}-0.012$ & $2.47 \times 10^{-4}-0.006$ & $(1.63-6.28) \times 10^{-3}$ & $(.402-2.06) \times 10^{-3}$ \\
$m_{\nu_{2}}$ & $0.0084-0.0149$ & $0.0084-0.0108$ & $0.0086-0.0113$ & $0.0084-0.0096$ \\
$m_{\nu_{3}}$ & $0.0456-0.0577$ & $0.0455-0.0575$ & $0.0446-0.0576$ & $0.0455-0.0573$ \\
$\theta_{12}$ & $29.30^{\circ}-39.20^{\circ}$ & $29.30^{\circ}-39.20^{\circ}$ & $29.30^{\circ}-39.20^{\circ}$ & $29.30^{\circ}-39.04^{\circ}$ \\
$\theta_{23}$ & $35.70^{\circ}-55.60^{\circ}$ & $35.70^{\circ}-55.60^{\circ}$ & $35.70^{\circ}-55.59^{\circ}$ & $35.70^{\circ}-40.15^{\circ}$ \\
$\theta_{13}$ & $0.084^{\circ}-11.50^{\circ}$ & $1.14^{\circ}-11.50^{\circ}$ & $3.60^{\circ}-11.15^{\circ}$ & $8.43^{\circ}-11.50^{\circ}$ \\
$J$ & $-0.0462-.0448$ & $-0.0459-.0463$ & $\sim 0$ & $\sim 0$ \\
$\delta$ & $-90^{\circ}-90.0^{\circ}$ & $-90^{\circ}-90.0^{\circ}$ & $\sim 0^{\circ}$ & $\sim 0^{\circ}$ \\
$\left\langle m_{e e}\right\rangle$ & - & $.00086-.0173$ & - & $.0032-.0075$ \\
\hline
\end{tabular}

Table 1: Calculated ranges for neutrino mass and mixing parameters obtained by varying $\phi_{1}$ and $\phi_{2}$ from 0 to $2 \pi$ for the normal hierarchy case. Inputs have been defined in the text. All masses are in $\mathrm{eV}$.

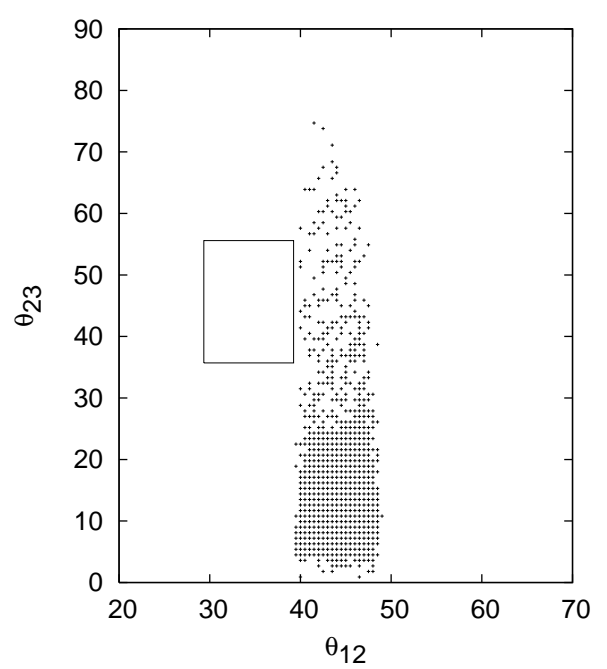

(a)

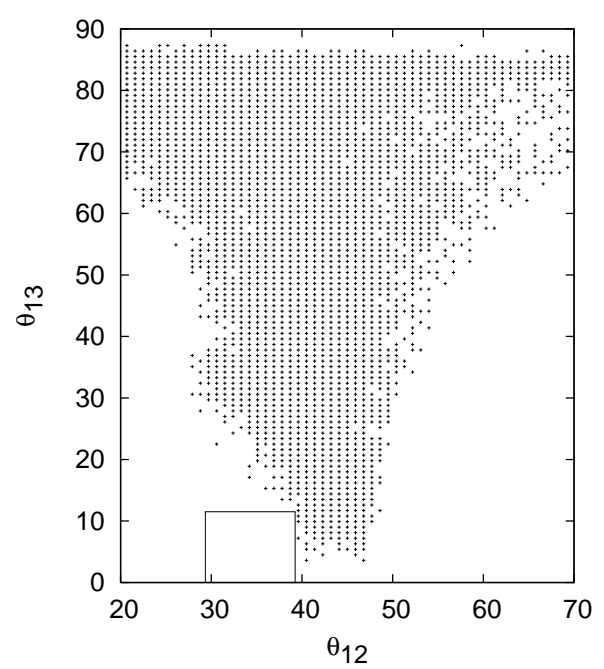

(b)

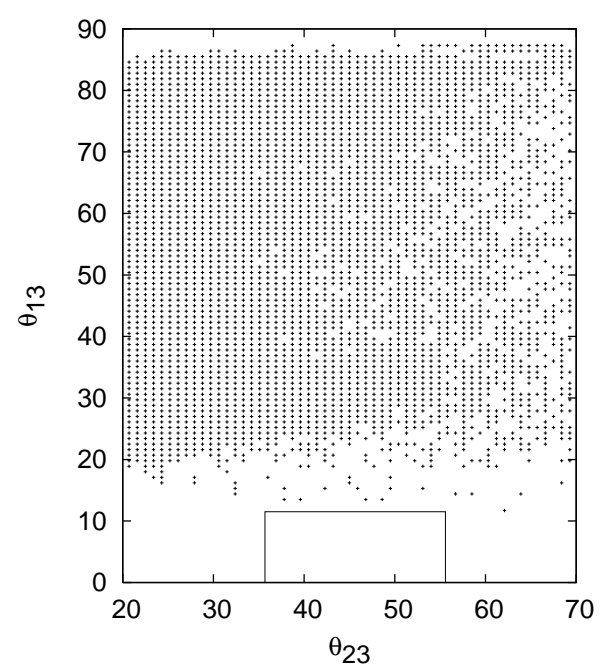

(c)

Figure 1: Plots showing the parameter space corresponding to any of the two mixing angles by constraining the third angle by its experimental limits given in equation (22) and giving full allowed variation to other parameters for Majorana neutrinos. The blank rectangular region indicates the experimentally allowed $3 \sigma$ region of the plotted angles. 


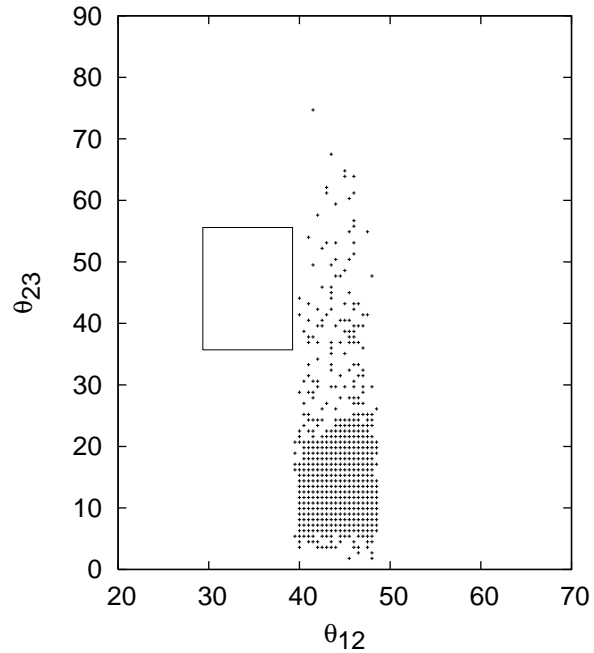

(a)

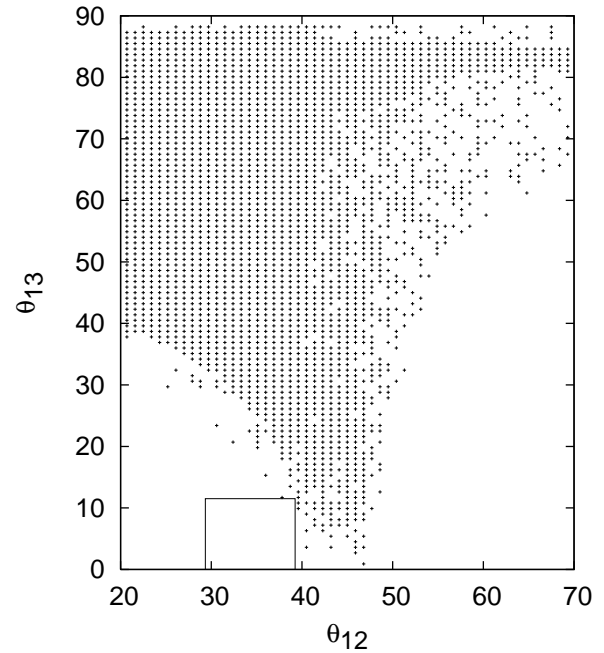

(b)

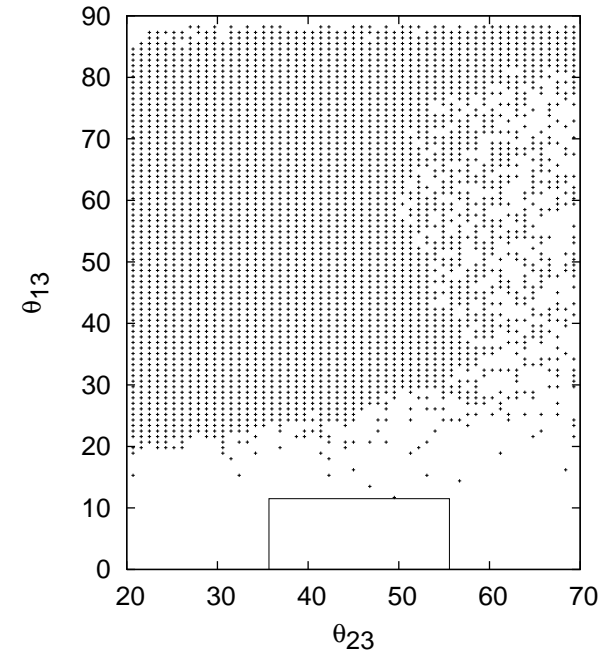

(c)

Figure 2: Plots showing the parameter space corresponding to any of the two mixing angles by constraining the third angle by its experimental limits given in equation (22) and giving full allowed variation to other parameters for Dirac neutrinos. The blank rectangular region indicates the experimentally allowed $3 \sigma$ region of the plotted angles.

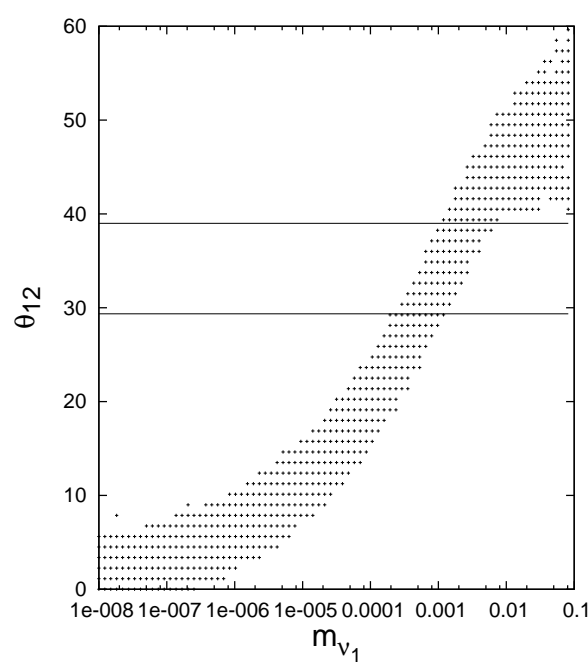

(a)

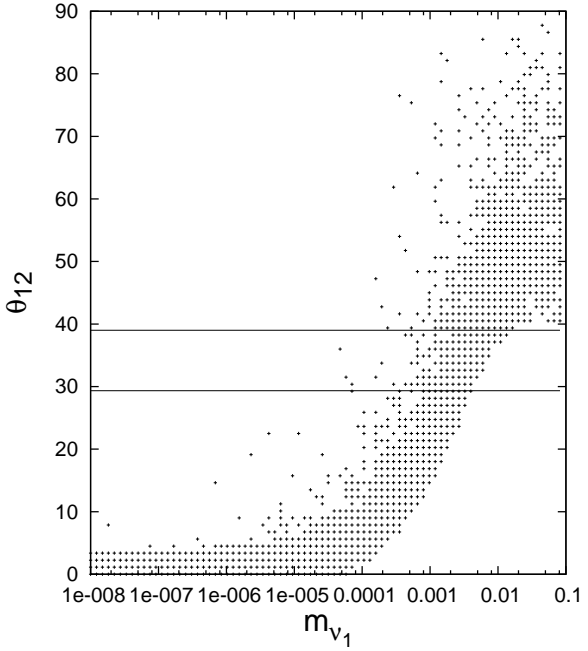

(b)

Figure 3: Plots showing variation of mixing angle $\theta_{12}$ with lightest neutrino mass $m_{\nu_{1}}$ by giving full variation to other parameters for neutrinos being (a) Majorana-like and (b) Dirac-like. The parallel lines indicate the $3 \sigma$ limits of angle $\theta_{12}$. 